Mathematical Research Letters 2, 9-13 (1995)

\title{
MORE CONSTRAINTS ON SYMPLECTIC FORMS FROM SEIBERG-WITTEN INVARIANTS
}

\author{
Clifford Henry Taubes
}

Recently, Seiberg and Witten (see [SW1], [SW2], [W]) introduced a remarkable new equation which gives differential-topological invariants for a compact, oriented 4-manifold with a distinguished integral cohomology class which reduces $\bmod (2)$ to the 2 nd Steiffel-Whitney class of the manifold. A brief mathematical description of these new invariants is given in the recent preprint [KM1].

Using the Seiberg-Witten equations, I proved in [T] the following:

Theorem 1. Let $X$ be a compact, oriented, 4 dimensional manifold with $b^{2+} \geq 2$. Let $\omega$ be a symplectic form on $X$ with $\omega \wedge \omega$ giving the orientation. Then the first Chern class of the canonical bundle of a compatible, almost complex structure on $X$ has Seiberg-Witten invariant equal to \pm 1 .

(A corollary of this theorem is the assertion that connect sums of nonnegative definite compact, oriented 4-manifolds do not admit symplectic forms which are compatible with the orientation.)

Subsequently, I have found that a slight modification of the proof of Theorem 1 gives further results about symplectic 4-manifolds. The purpose of this note is to report on these additional results.

The first result below constrains the other cohomology classes on $X$ which have non-zero Seiberg-Witten invariant. In the theorem below, $[\omega]$ denotes the cohomology class of the symplectic form $\omega$, and $K \rightarrow X$ is the canonical bundle for any almost complex structure on $X$ which is compatible with $\omega$. Also, the symbol $\bullet$ denotes the bilinear pairing on cohomology as given by cup product and evaluation on the fundamental class of $X$.

Theorem 2. Let $X$ be a compact, oriented symplectic manifold with $b^{2+} \geq$ 2 and with symplectic form $\omega$ which is compatible with the given orientation. Let $c \in H^{2}(X ; \mathbb{Z})$ have non-zero Seiberg-Witten invariant. Then

Received November 22, 1994.

Research supported in part by the National Science Foundation. 
$|c \bullet[\omega]| \leq c_{1}(K) \bullet[\omega]$ and if equality holds then either $\pm c$ is equal to $c_{1}(K)$. In particular, if $X$ is to admit a symplectic form, then $c_{1}(K) \bullet[\omega] \geq 0$.

Theorem 2 is proved in [W] for Kähler manifolds with $b^{2} \geq 3$. (The assertion that $c_{1}(K) \bullet[\omega] \geq 0$ for Kähler manifolds with $b^{2} \geq 2$ follows from the fact that such manifolds have $\left(b^{2+}-1\right) / 2$ holomorphic sections of K.)

There is another proof of Theorem 2 which uses a result recently announced by Donaldson concerning the existence of symplectic submanifolds of a symplectic manifold. Using Donaldson's existence assertion, Theorem 2 follows with a proof of an adjunction type formula for the line bundles with non-zero Seiberg-Witten classes. (Donaldson has also noted the other proof.) The aforementioned adjunction formula for the Seiberg-Witten classes is the analog of a formula proved by Kronheimer and Mrowka [KM2] for their basic class description of Donaldson's polynomial. A version of the Seiberg-Witten adjunction formula is proved in [KM1] and the general version will be discussed in a separate paper with other authors.

Another variant of the proof of Theorem 1 yields

Theorem 3. The manifold $\mathbb{C P}^{2}$ has no symplectic form $\omega$ for which $c_{1}(K) \bullet[\omega]>0$. ( The standard Kähler structure on $\mathbb{C P}^{2}$ has $c_{1}(K) \bullet[\omega]<0$.)

Note that the inequalities for $c_{1}(K) \bullet[\omega]$ in Theorems 2 and 3 go in opposite ways. But, there is no contradiction here because $\mathbb{C P}^{2}$ has $b^{2+}=1$.

\section{a) Proof of Theorem 2}

The reader should first become familiar with the proof of Theorem 1 in [T], for the proof of Theorem 2 will proceed almost verbatim as that of Theorem 1 modulo some minor changes in notation. To begin, one should fix a metric on $X$ for which the symplectic form $\omega$ is self dual. Then, the $\operatorname{spin}_{\mathbb{C}}$ bundle for $K^{-1}$ splits as $S_{+}=\mathbb{I} \oplus K^{-1}$ where the form $\omega$ acts (by Clifford multiplication) on the $\mathbb{I}$ summand as multiplication by $-i$ and on the other summand as multiplication by $i$. Remember that there is a unique connection $A_{0}$ on $K^{-1}$ which is such that the $\operatorname{spin}_{\mathbb{C}}$ covariant derivative $\widehat{\nabla} A_{0}$ induces the trivial covariant derivative $d$ on the $\mathbb{I}$ summand. (This induced covariant derivative is $2^{-1} \cdot(1+i \omega) \cdot \widehat{\nabla}_{A_{0}} \cdot 2^{-1} \cdot(1+i \omega)$.)

To prove Theorem 2, assume that there is a line bundle $L$ over $X$ whose first Chern class has non-zero Seiberg-Witten invariant which violates the conditions in the theorem. Such an assumption will be seen to lead directly to a contradiction. In deriving this contradiction, it is necessary to remark first that if $c_{1}(L)$ has non-zero Seiberg-Witten invariant, then so does $-c_{1}(L)$ (see $[\mathrm{W}]$ ). Thus, if Theorem 2's conditions are violated, they 
are violated by an $L$ with

$$
c_{1}(L) \bullet[\omega]+c_{1}(K) \bullet[\omega] \leq 0 .
$$

The line bundle $L$ can be written as $K^{-1} \otimes E^{2}$, where $E \rightarrow X$ is another complex line bundle. With this understood, the $\operatorname{spin}_{\mathbb{C}}$ spinors for $L$ decompose as $S_{L+}=E \oplus\left(E \otimes K^{-1}\right)$ and a spinor $\psi$ will be written as $\left(\alpha \cdot u_{0}, \beta\right)$ where $\alpha$ is a section of $E$ and $\beta$ one of $E \otimes K^{-1}$. Here $u_{0}$ is (as in $[\mathrm{T}])$ the unit length, $A_{0}$-covariantly constant section of the summand $\mathbb{I}$ in $\mathbb{I} \oplus K^{-1}$. A choice of connection $A$ on the line bundle $L$ gives a $\operatorname{spin}_{\mathbb{C}}$ covariant derivative $\widehat{\nabla}_{A}$ on $S_{L+}$. This $\widehat{\nabla}_{A}$ induces covariant derivatives on the two summands of $S_{L+}$. These induced covariant derivatives are written as:

$$
\begin{aligned}
& 2^{-1} \cdot(1+i \omega) \cdot \widehat{\nabla}_{A}\left(\alpha u_{0}\right) \equiv\left(\nabla_{a} \alpha\right) \cdot u_{0}, \\
& \text { 2) } 2^{-1} \cdot(1-i \omega) \cdot \widehat{\nabla}_{A} \beta \equiv \nabla_{A}^{\prime} \beta .
\end{aligned}
$$

Here, $\nabla_{a}$ is a covariant derivative on $E$ and $\nabla_{A}^{\prime}$ is one on $E \otimes K^{-1}$.

With these preliminaries out of the way, consider now the perturbed Seiberg-Witten equation in (6) of [T] with the parameter $r \in[0, \infty)$ as an equation for a connection $A$ on $L$ and a section $\psi=\left(\alpha u_{0}, \beta\right)$ of $S_{L+}$ :

$$
\begin{gathered}
D_{A} \psi=0 \\
P_{+} F_{A}=P_{+} F_{A_{0}}+i \cdot\left(|\alpha|^{2}-|\beta|^{2}-1\right) \cdot \omega-i \cdot\left(\alpha^{*} \beta+\alpha \beta^{*}\right) \\
-i \cdot 4 \cdot r \cdot\left(1+r \cdot|\alpha|^{2}\right)^{-1} \cdot\left(\alpha^{*}\left\langle b, \nabla_{a} \alpha\right\rangle-\alpha \cdot\left\langle b, \nabla_{a} \alpha\right\rangle^{*}\right) .
\end{gathered}
$$

Here, $b$ is the section of $K^{-1} \otimes T^{*} X_{\mathbb{C}}$ which is defined (as in (1) in [T]) by the equation $\widehat{\nabla}_{A_{0}} u_{0}=b$. (In $(3), D_{A}$ denotes the $\operatorname{spin}_{\mathbb{C}}$ Dirac operator on $S_{L+}$ as defined by the Riemannian metric and $A$.)

Lemmas 2, 3 in [T] have essentially verbatim analogs for this $L$ version of the perturbed Seiberg-Witten equation. Also, because of (1), the $L$ version of Lemma 4 in $[\mathrm{T}]$ also holds. Thus, Proposition 5 in [T] also has a selfevident $L$ version. Consider now whether one can prove the $L$ analog of Lemma 6 in $[T]$. Proceeding as in Step 1 of Section c of $[T]$, one finds the $L$-analog of Lemma 8 by directly copying the arguments in [T]. Likewise, the $L$ version of (30) in [T] also holds, but the $L$ version of (31) in [T] may not. Instead, one has

$$
\begin{gathered}
\int\left(\left|\nabla_{a} \alpha\right|^{2}+\left(|\alpha|^{2}-1-|\beta|^{2}\right)\left(|\alpha|^{2}-1\right)\right)-\int\left(1-|\alpha|^{2}-|\beta|^{2}\right) \\
=\int\left\langle D_{A}\left(\alpha u_{0}\right), D_{A} \beta\right\rangle .
\end{gathered}
$$


In the case where $L=K^{-1}$, the second integral on the right side above is zero. In general, this integral can be identified using (3) as equal to $2 \pi$ times the left hand side of (1). Thus, in the case where (1) holds, one has

$$
\int\left(\left|\nabla_{a} \alpha\right|^{2}+\left(|\alpha|^{2}-1-|\beta|^{2}\right)\left(|\alpha|^{2}-1\right)\right) \leq \int\left\langle D_{A}\left(\alpha u_{0}\right), D_{A} \beta\right\rangle
$$

which implies that (32) in [T] holds for the $L$-version of (5).

The remaining steps in the proof of Theorem 1 of $[\mathrm{T}]$ can then be carried out through to the end. (These only involve integrations by parts, the triangle inequality and a standard Sobolev inequality.) These steps lead directly to a contradiction; namely that $|\alpha| \equiv 1$ and that $\nabla_{a} \alpha \equiv 0$. To avoid this contradiction, one is inescapably forced to conclude that (1) can't be negative; and if (1) is equal to zero, then $L=K^{-1}$.

\section{b) Proof of Theorem 3}

Suppose that $\omega$ is a symplectic form on $\mathbb{C P}^{2}$ and let $K$ denote the canonical bundle for a compatible almost complex structure. The arguments in [T] for the proof of Theorem 1 show that the perturbed Seiberg-Witten equation (3) has a unique solution up to gauge $\left(A_{0}, u_{0}\right)$ for all $r$ sufficiently large. Lemma 4 in $[\mathrm{T}]$ holds, and this means that the $r=0$ version of (3) computes a Seiberg-Witten invariant of \pm 1 for $K^{-1}$. But, note that $\mathbb{C P}^{2}$ has a metric with positive scalar curvature, and this means that the Seiberg-Witten invariant for $K^{-1}$ is zero [W] when computed by Seiberg and Witten's original equation

$$
\begin{gathered}
D_{A} \psi=0, \\
P_{+} F_{A}=i \cdot\left(|\alpha|^{2}-|\beta|^{2}\right) \cdot \omega-i \cdot\left(\alpha^{*} \beta+\alpha \beta^{*}\right) .
\end{gathered}
$$

The disagreement between the $r=0$ version of (3) and (6) leads directly to Theorem 3. Indeed, consider the family of equations below as parameterized by $s \in[0,1]$ :

$$
\begin{gathered}
D_{A} \psi=0, \\
P_{+} F_{A}=s \cdot P_{+} F_{A_{0}}+i \cdot\left(|\alpha|^{2}-|\beta|^{2}-s\right) \cdot \omega-i \cdot\left(\alpha^{*} \beta+\alpha \beta^{*}\right) .
\end{gathered}
$$

The $s=1$ version of (7) is the $r=0$ version of (3), and the $s=0$ version of (7) is (6). The only way (3) and (6) can disagree on the Seiberg-Witten invariant of $K^{-1}$ is if there exists some $s \in[0,1]$ for which $(7)$ has a $\psi \equiv 0$ solution. (The vernacular for this is that there is a "wall crossing" for some value of the parameter $s$.) If a solution $(A, 0)$ solves $(7)$ for some particular 
$s \in[0,1]$, then wedge both sides of (7) with the symplectic form $\omega$ and integrate over $\mathbb{C P}^{2}$ to conclude that

$$
c_{1}\left(K^{-1}\right) \bullet[\omega]=s \cdot c_{1}\left(K^{-1}\right) \bullet[\omega]+s \cdot[\omega] \bullet[\omega] .
$$

This last possiblity is forbidden if $c_{1}\left(K^{-1}\right) \bullet[\omega]<0$. (Note that (8) will occur for some $s$ if $c_{1}\left(K^{-1}\right) \bullet[\omega]>0$ as happens with the standard Kähler structure on $\mathbb{C P}^{2}$.)

\section{References}

[KM1] P. Kronheimer and T. Mrowka, The genus of embedded surfaces in the projective plane, Math. Res. Letters 1 (1994), 797-808.

[KM2] - Recurrence relations and asymptotics for four-manifold invariants, Bull. Amer. Math. Soc. (to appear).

[SW1] N. Seiberg and E. Witten, Electric-magnetic duality, monopole condensation, and confinement in $N=2$ supersymmetric Yang-Mills theory, (preprint).

[SW2] - Monopoles, duality and chiral symmetry breaking in $N=2$ supersymmetric $Q C D$, (preprint).

[T] C. H. Taubes, The Seiberg-Witten invariants and symplectic forms, Math. Res. Letters 1 (1994), 809-822.

[W] E. Witten, Lectures at MIT and Harvard, Fall 1994; and Monopoles and fourmanifolds, Math. Res. Letters 1 (1994), 769-796.

Department of Mathematics, Harvard University, Cambridge, M a 02138

E-mail address: chtaubes@math.harvard.edu 